\title{
ON THE ESSENTIAL ALGEBRAIC ASPECTS OF SUBMANIFOLD QUANTUM MECHANICS
}

\author{
SHIGEKI MATSUTANI
}

Communicated by Boris Konopeltchenko

\begin{abstract}
The submanifold quantum mechanics was opened by Jensen and Koppe and has been studied for more than three decades. This article gives its more algebraic definition and show the essential aspects of the submanifold quantum mechanics from an algebraic viewpoint.
\end{abstract}

\section{Introduction}

The submanifold quantum mechanics was initiated by Jensen and Koppe [18] and da Costa [8] and has been developed further by Duclos, Exner, Krejčiřík, Šeba and S̆tovíček [11, 10, 13, 19], Ikegami, Nagaoka, Takagi and Tanzawa [16, 17, 33], Clark and Bracken [6, 7], Goldstone and Jaffe [14], Burgess and Jensen [5], Encinosa and Etemadi [12], Mladenov [29], Suzuki, Tsuru and the present author [20-26, 28, 30-32]. In all these theories, we obtain differential operators over a submanifold $S$ in an Euclidean space relying on a confinement potential and taking some squeezing limit of the potential.

The so obtained differential operators however do not strongly depend upon the shape of confinement potentials or in the way the squeezing limit is taken. Further they exhibit geometrical nature of the submanifold. In fact, the Dirac operators obtained in the scheme are related to the Frenet-Serret and the generalized Weierstrass relations [21-28] from which we can recover all geometrical data of the submanifold. Thus we believe that they should be obtained beyond any approximation and defined more algebraically.

In this article, we will give a more algebraic definition of the submanifold quantum mechanics, which is free from any approximation theories, and show what is the essential of the submanifold quantum mechanics from an algebraic point of view. 


\section{Self-adjoint Operators}

As will be shown later, in submanifold quantum mechanics, a non-unitary transformation plays a key role, which converts a not self-adjoint operator into selfadjoint. Thus as a preparation, we should investigate the self-adjoint operators more precisely. However the concept of "adjointness" of an operator is subtle even in the study of algebra of differential operators as in [Remark 1.2.16 in [3]]. Let us consider a differential operator $\partial / \partial z^{\alpha}$ defined over a $n$-dimensional differentiable manifold $M$ with local coordinate $s\left(z_{1} . z_{2}, \ldots, z_{M}\right)$. Let $\partial / \partial z^{\alpha}$ acts on the functions from left hand side conventionally. Provided that $M$ is equipped with a metric $g$ and a volume form $g^{1 / 2} \mathrm{~d}^{n} z$, we have a natural pairing for smooth wavefunctions $f_{1}$ and $f_{2}$ whose support is compact

$$
\left\langle f_{1} \mid f_{2}\right\rangle_{g}=\int_{M} g^{1 / 2} f_{1}^{*}(z) f_{2}(z) \mathrm{d}^{n} z .
$$

Using this pairing, an expectation value of $\partial / \partial z^{\alpha}$ is also naturally defined by

$$
\left\langle f_{1} \mid \frac{\partial}{\partial z^{\alpha}} f_{2}\right\rangle_{g}=\int_{M} g^{1 / 2} f_{1}^{*}(z) \frac{\partial}{\partial z^{\alpha}} f_{2}(z) \mathrm{d}^{n} z .
$$

The adjoint operator of $\frac{\partial}{\partial z^{\alpha}}$ is given as $\left(\frac{\partial}{\partial z^{\alpha}}\right)^{*}=-\frac{\partial}{\partial z^{\alpha}}-\frac{1}{2}\left(\frac{\partial \log g}{\partial z^{\alpha}}\right)$, which obviously depends upon the measure. For another measure $g^{1 / 2} \mathrm{~d}^{n} z$ such that $g^{1 / 2} \mathrm{~d}^{n} z f_{1}^{*}(z) f_{2}(z)=g^{\prime 1 / 2} \mathrm{~d}^{n} z\left(\sigma f_{1}\right)^{*}(z)\left(\sigma f_{2}\right)(z)$ where $\sigma:=\left(g / g^{\prime}\right)^{1 / 4}$, we may have another expectation value

$$
\left\langle f_{1} \mid \frac{\partial}{\partial z^{\alpha}} f_{2}\right\rangle_{g^{\prime}}=\int_{M} g^{\prime 1 / 2} f_{1}^{*}(z) \frac{\partial}{\partial z^{\alpha}} f_{2}(z) \mathrm{d}^{n} z
$$

and a different adjoint operator $\left(\partial / \partial z^{\alpha}\right)^{*}$. The adjointness has such an ambiguity. Using this ambiguity, we can introduce the half-density and then any $\partial / \partial z^{\alpha}$ can be self-adjoint by setting $g^{\prime}=1$ (Theorem 18.1.34 in [15]). However in general the measure $g^{1 / 2} \mathrm{~d}^{n} z$, e.g., Haar measure, exhibits a geometrical nature of the space. Thus the measureless expression such as the half-density sometimes are less effective. For example, in the theory of the ordinary second order differential equation related to orthonormal polynomial functions, the concept of the halfdensity implicitly appears (p. 424 in [2]) but gives only very rough estimations, such as the asymptotic expressions of the functions. On the other hand, their 
expressions with the proper measure as the orthonormal polynomials give more precise information.

In the submanifold quantum mechanics $[8,18]$, we partially use the half-density in the framework of a theory with the Haar measure as we will show later.

From quantum mechanical point of view, the first problem in quantum mechanics over a curved system is to search for a proper metric and a proper measure. This problem is easily solved for quantum mechanics over a curved object in the Euclidean space $\mathbb{E}^{3}$. In $\mathbb{E}^{3}$, the ordinary Lebesgue measure is natural because it is the Haar measure for the translations. As the quantum mechanics is established in $\mathbb{E}^{3}$ and the concept of the adjoint operator plays essential role [9], we will use the induced metric on the curved object from that one in $\mathbb{E}^{3}$.

Next we will review the properties of a self-adjoint operators precisely. We will deal with the space of functions $\Omega$ and its dual $\Omega^{*}$ with $\mathrm{L}^{2}$-type paring $\langle$,$\rangle :$ $\Omega^{*} \times \Omega \rightarrow \mathbb{C}$ using the Lebesgue measure in $\mathbb{E}^{3}$. Let us consider an operator $Q$ which domain is $\Omega$. Then if exists, we will define a right-adjoint operator, $\operatorname{Ad}(Q)$, with the domain $\Omega^{*}$ by

$$
\langle f, Q g\rangle=\langle f \operatorname{Ad}(Q), g\rangle, \quad \text { for }(f, g) \in \Omega^{*} \times \Omega .
$$

Assume that there is an isomorphism $\varphi$ between $\Omega$ and $\Omega^{*}$ as vector spaces. Then the triplet $\left(\Omega^{*} \times \Omega,\langle\rangle,, \varphi\right)$ becomes a Hilbert space $\mathcal{H}$ by introducing the inner product $():, \Omega \times \Omega \rightarrow \mathbb{C}$ with $(f, g):=\langle\varphi(f), g\rangle$ after completion in $($,$) . We$ assume such completion in this article and thus we write $\left(\Omega^{*} \times \Omega,\langle\rangle,, \varphi\right)=\mathcal{H}$ hereafter. Then the ordinary adjoint operator $Q^{*},\left(Q^{*} f, g\right)=(f, Q g)$, is given by $Q^{*} f:=\varphi^{-1}(\varphi(f) \operatorname{Ad}(Q))$. In general, the dual space $\Omega^{*}$ of $\Omega$ is greater than $\Omega$ itself and we regard $\Omega^{*}$ as the image of $\varphi$. In this construction, the Dirac $\delta$ functions are elements in the complement of the image of $\varphi$ in the dual space.

Suppose that the operator $Q$ is self-adjoint, i.e., the domains of $Q^{*}$ and $Q$ coincide and $Q^{*}=Q$ there. Then we have the following properties:

1. The kernel of $Q, \operatorname{ker}(Q)$, is isomorphic to the $\operatorname{ker}(\operatorname{Ad}(Q))$, i.e.,

$$
(\operatorname{ker}(Q))^{*}:=\varphi(\operatorname{ker}(Q))=\operatorname{ker}(\operatorname{Ad}(Q))
$$

2. The projection $\pi_{Q}$ from $\Omega^{*} \times \Omega$ to $(\operatorname{ker}(Q))^{*} \times \operatorname{ker}(Q)$ is commutative with $\varphi$, i.e.,

$$
\varphi \pi_{Q \mid \Omega}=\pi_{Q \mid \Omega^{*}} \varphi, \quad\left(\varphi\left(\pi_{Q \mid \Omega f}\right)=\pi_{Q \mid \Omega^{*}} \varphi(f) \equiv \varphi(f) \operatorname{Ad}\left(\pi_{Q \mid \Omega}\right)\right)
$$

3. $\left((\operatorname{ker}(Q))^{*} \times \operatorname{ker}(Q),\langle\rangle,, \varphi\right)$ becomes a Hilbert space. 
For $\pi_{Q}$ satisfying (5), we will say that $\pi_{Q}$ is consistent with the inner product. In fact (5) means that $\varpi_{Q}:=\left.\pi_{Q}\right|_{\Omega}$ is a projection operator as $*$-algebra [1]: $\varpi_{Q}^{2}=$ $\varpi_{Q}$ and $\varpi_{Q}^{*}=\varpi_{Q}$ due to the relation $\varpi_{Q}^{*} f \equiv \varphi^{-1}\left(\varphi(f) \operatorname{Ad}\left(\varpi_{Q}\right)\right)=\varpi_{Q} f$.

We will end the review of the properties of self-adjoint operators by giving a physical setting for the submanifold quantum mechanics. Though we can do this in more general situation, we will investigate only the case of a surface in the three dimensional Euclidean space $\mathbb{E}^{3}$.

\section{Geometry of an Embedded Surface}

For a smooth surface $S$ embedded in the euclidean space $\mathbb{E}^{3}$ with the metric induced on $S$ from the metric on $\mathbb{E}^{3}$, we consider a Schrödinger equation over a tubular neighborhood $T_{S}$ of $S, \pi_{S}: T_{S} \rightarrow S$, with the $\mathrm{L}^{2}$-type Hilbert space $\mathcal{H}=\left(\Omega^{*} \times \Omega,\langle,\rangle_{G_{S}}, \varphi\right)$

$$
-\Delta \psi=E \psi \quad \text { over } \quad T_{S}
$$

Here $\Omega^{*}$ and $\Omega$ consist of smooth compact support functions over $T_{S}$ and $\Delta$ is the Laplacian in $\mathbb{E}^{3}$. Following $[8,18]$, we will add a potential to the left hand side in (6), which confines a particle in the tubular neighborhood $T_{S}$. This potential restrains the support of the wavefunctions in $T_{S}$. By taking a squeezing limit of the potential, we decompose the system in the normal and the tangential modes, suppress the normal mode, and obtain the submanifold Schrödinger equation along the surface $S$. Instead of the above scheme, below we will provide another definition of the Schrödinger operator specified by (16).

Before giving the new definition, we will present a geometrical preliminary. Let $S$ be locally expressed by a coordinate system $\left(s^{1}, s^{2}\right)$ and $q$ be a normal coordinate of $T_{S}$ whose absolute value is the distance from the surface $S, \mathrm{~d} q$ is an infinitesimal length in $\mathbb{E}^{3}$ and belongs to the kernel of $\pi_{S *}$ and $\mathrm{d} q\left(\partial_{\alpha}\right)=0$ $\left(\alpha=1,2, \partial_{\alpha}:=\partial / \partial s^{\alpha}\right)$. A point in $T_{S}$ expressed by the affine coordinate $\mathbf{x}:=\left(x^{1}, x^{2}, x^{3}\right)$ in $\mathbb{E}^{3}$ can be uniquely represented by

$$
\mathbf{x}=\pi_{S} \mathbf{x}+q \mathbf{e}_{3}
$$

where $\mathbf{e}_{3}$ is the normal unit vector at $S$. The moving frame $E_{\alpha}^{i}=\partial_{\mu} x^{i},(\mu=$ $1,2,3, i=1,2,3)$ is written by

$$
E_{\alpha}^{i}=e_{\alpha}^{i}+q^{3} \gamma_{3 \alpha}^{\beta} e_{\beta}^{i}, \quad E_{3}^{i}=e_{3}^{i}
$$

where $\alpha, \beta=1,2, e_{\alpha}^{i}:=\partial_{\alpha}\left(\pi_{S} x^{i}\right)$, and

$$
\partial_{\alpha} \mathbf{e}_{3}=\gamma_{3 \alpha}^{\beta} \mathbf{e}_{\beta}
$$


Thus the metric, $G_{S \mu \nu}:=\delta_{i j} E_{\mu}^{i} E_{\nu}^{j},(\mu, \nu=1,2,3)$, induced from that one in the Euclidean space $\mathbb{E}^{3}$ is given as

$$
\begin{gathered}
G_{S}=\left(\begin{array}{cc}
g_{S}(q) & 0 \\
0 & 1
\end{array}\right) \\
g_{S}(q)_{\alpha \beta}=g_{S \alpha \beta}+\left[\gamma_{3 \alpha}^{\gamma} g_{S \gamma \beta}+g_{S \alpha \gamma} \gamma_{3 \beta}^{\gamma}\right] q+\left[\gamma_{3 \alpha}^{\delta} g_{S \delta \gamma} \gamma_{3 \beta}^{\gamma}\right] q^{2}
\end{gathered}
$$

where $g_{S \alpha \beta}:=\delta_{i j} e_{\alpha}^{i} e_{\beta}^{j}$. The determinant of the metric is expressed as

$$
\operatorname{det} G_{S}=\rho \operatorname{det} g_{S}, \quad \rho=1+\operatorname{tr}\left(\gamma_{3 \beta}^{\alpha}\right) q+\operatorname{det}\left(\gamma_{3 \beta}^{\alpha}\right) q^{2}
$$

and thus the pairing $\langle,\rangle_{G_{S}}$ is expressed by

$$
\left\langle\psi_{1}, \psi_{2}\right\rangle_{G_{S}}=\int\left(\operatorname{det} g_{S}\right)^{1 / 2} \rho^{1 / 2} \psi_{1}^{*} \psi_{2} \mathrm{~d}^{2} s \mathrm{~d} q .
$$

Following $[8,18]$, we wish to establish the quantum mechanics of a particle restricted to $S \subset \mathbb{E}^{3}$. The restriction of the particle to the surface requires that the components of momentum and position of the particle in the normal direction vanish. We have the canonical commutation relation for the normal direction, $\left[\sqrt{-1} \partial_{q}, q\right] \equiv \sqrt{-1} \partial_{q} q-q \sqrt{-1} \partial_{q}=\sqrt{-1}$ and wish to consider the kernel of $p_{q}:=\sqrt{-1} \partial_{q}$. Let us note that for a function $h(q)$ of $q,[h(q), q]=0$ and thus we have $\left[\sqrt{-1} \partial_{q}+h(q), q\right]=\sqrt{-1}$. Using this ambiguity, we can optimize $h$ so that $\sqrt{-1} \partial_{q}+h(q)$ is self-adjoint. However $p_{q}$ is not self-adjoint in general due to the existence of $\rho$ in (12) and thus a projection to the kernel of $\partial_{q}$ will be not consistent with the inner product in the above sense and we will give another equivalent scheme called self-adjointization.

Thus we deform the Hilbert space so that $p_{q}$ becomes a self-adjoint operator by using the half-density theory (Theorem 18.1.34 in [15]). As we will show (see (13) and (14) below), there exist a Hilbert space $\mathcal{H}^{\prime} \equiv\left(\tilde{\Omega}^{*} \times \tilde{\Omega},\langle,\rangle_{S}, \tilde{\varphi}\right)$ and self-adjointization: $\eta_{\mathrm{sa}}: \mathcal{H} \rightarrow \mathcal{H}^{\prime}$ satisfying the following properties

1) There exists an isomorphism between $\Omega^{*} \times \Omega \rightarrow \tilde{\Omega}^{*} \times \tilde{\Omega}$ as vector spaces. We denote it by the same $\eta_{\text {sa }}$

2) By defining a pairing $\langle\circ, \times\rangle_{g_{S}}:=\left\langle\eta_{\mathrm{sa}} \circ, \eta_{\mathrm{sa}} \times\right\rangle_{G_{S}}$, we set $\tilde{\varphi}:=\eta_{\mathrm{sa}} \varphi \eta_{\mathrm{sa}}^{-1}$

3) An operator $Q$ in $\mathcal{H}$ is transformed into $\eta_{\mathrm{sa}}(Q)=\eta_{\mathrm{sa}} Q \eta_{\mathrm{sa}}^{-1}$

4) $p_{q}$ itself (not $\eta_{\mathrm{sa}}\left(p_{q}\right)$ ) becomes a self-adjoint operator in $\mathcal{H}^{\prime}$.

Of course, the self-adjointization is not a unitary operation and due to this operation, the inner product changes from $\langle,\rangle_{G_{S}}$ to $\langle,\rangle_{g_{S}}$. 
Using the dependence of the adjoint operator upon the measure as mentioned above, the self-adjointization $\eta_{\mathrm{sa}}$ is realized as follows

$$
\begin{gathered}
\eta_{\mathrm{sa}}\left(\psi_{1}^{*}\right)=\rho^{-1 / 4} \psi_{1}^{*}, \quad \eta_{\mathrm{sa}}\left(\psi_{2}\right)=\rho^{-1 / 4} \psi_{2}, \quad \eta_{\mathrm{sa}}(\Delta)=\rho^{-1 / 4} \Delta \rho^{1 / 4} \\
\left\langle\psi_{1}, \psi_{2}\right\rangle_{g_{S}}=\int\left(\operatorname{det} g_{S}\right)^{1 / 2} \psi_{1}^{*} \psi_{2} \mathrm{~d}^{2} s \mathrm{~d} q .
\end{gathered}
$$

In $T_{S}, \rho$ does not vanish and therefore (13) defines an isomorphisms. Since the measure in (14) is $q$-independet, $p_{q}:=\sqrt{-1} \partial_{q}$ itself becomes a self-adjoint operator in $\mathcal{H}^{\prime}$. Here we should notice that the proper treatment of the self-adjoint operators and a prototype of self-adjointization appear implicitly in the study of hydrogen atom in the classical book by Dirac [9]. In the book, instead of $p_{q}$, the formally self-adjoint operator $\eta_{\mathrm{sa}}^{-1}\left(p_{q}\right)$ is treated which determines an additional term $h(q)$ to $\sqrt{-1} \partial_{q}$ so that it becomes self-adjoint. Corresponding to the property 4) of $\eta_{\mathrm{sa}}$, the transformation from $\sqrt{-1} \partial_{q}$ to $\eta_{\mathrm{sa}}^{-1}\left(p_{q}\right)$ is not unitary. Hence the projection

$$
\pi_{p_{q}}: \tilde{\Omega}^{*} \times \tilde{\Omega} \rightarrow\left(\operatorname{ker}\left(\operatorname{Ad}\left(p_{q}\right)\right)\right)^{*} \times \operatorname{ker}\left(p_{q}\right)
$$

is consistent with the inner product $($,$) . Due to the self-adjointness and the map$ $\tilde{\varphi}: \operatorname{ker}\left(p_{q}\right) \rightarrow \operatorname{ker}\left(\operatorname{Ad}\left(p_{q}\right)\right)$ the latter spaces are isomorphic. More precisely we should write $\tilde{\varphi}_{\mid \operatorname{ker}\left(p_{q}\right)}$ instead of $\tilde{\varphi}$.

In other words, $\mathcal{H}_{p_{q}}:=\left(\left(\operatorname{ker}\left(\operatorname{Ad}\left(p_{q}\right)\right)\right) \times \operatorname{ker}\left(p_{q}\right),(),, \tilde{\varphi}\right)$ presents a quantum mechanical system.

We should note that sometimes the Goldstone mode is given as a zero mode of a differential operator exhibiting a symmetry [4], i.e., $\psi \in \operatorname{ker}\left(p_{q}\right)$ behaves like a Goldstone mode for the normal translation mode. In fact, $p_{q}$ mode does not contribute to $\eta_{\mathrm{sa}}(\Delta)$ over the Hilbert space $\mathcal{H}_{p_{q}}$. The projection on $\mathcal{H}_{p_{q}}$ means vanishing momentum of the normal direction and kills the normal translation freedom. Hence we can choose a position $q$ as an ordinary symmetry breaking operator. Precisely speaking, since $\eta_{\mathrm{sa}}(\Delta)_{\mid \operatorname{ker}\left(p_{q}\right)}$ has $q$-dependence and the energy depends weakly upon $q$, it slightly differs from the ordinary symmetry breaking operator. However as the dependence is not strong, it can be justified. If we choose $q=q_{0}$ for all points in $S$, we have submanifold Schrödinger equation over a surface $S\left(q_{0}\right)$ given by $q=q_{0}$ instead of the surface $S$ for $q=0$. After choosing it as $q=0$, the Laplacian in (6) becomes

$$
\Delta_{S \hookrightarrow \mathbb{E}^{n}}:=\eta_{\mathrm{sa}}(\Delta)_{\left|\operatorname{ker}\left(p_{q}\right)\right| q=0}
$$

as an operator in $\mathcal{H}_{p_{q} \mid q=0}$. The first restriction ${ }_{\mid \operatorname{ker}_{q}}$ means the restriction of domain as an operator and the second one $\left.\right|_{\mid q=0}$ should be regarded as a restriction in 
the meaning of presheaf theory. Since (16) can be written by

$$
\Delta_{S \hookrightarrow \mathbb{E}^{n}}:=\left(\rho^{1 / 4} \Delta \rho^{-1 / 4}\right) \partial_{q}=0, q=0
$$

it might be expected that the definition should be expressed in differential ring theory [3]. By letting $K$ and $H$ denote the Gauss and mean curvatures of $S \subset \mathbb{E}^{3}$, we obtain the well-known operator $[8,18]$

$$
\Delta_{S \hookrightarrow \mathbb{E}^{3}}=\Delta_{S}+H^{2}-K
$$

and the submanifold Schrödinger equation

$$
-\Delta_{S \hookrightarrow \mathbb{E}^{3}} \psi=E \psi \quad \text { over } S
$$

in the Hilbert space $\mathcal{H}_{p_{q} \mid q=0}$. Here $\Delta_{S}$ is the Beltrami-Laplace operator on $S$ which exhibits the intrinsic properties of the surface $S$, whereas the second and the third terms in (17) represent the extrinsic properties of $S \subset \mathbb{E}^{3}$.

\section{Conclusions}

Here we emphasize that the definition (16) is very algebraic. Particularly in this construction, we did not use any approximation theories nor limit-theorems. Physically speaking, the above requirement of vanishing momentum and position will contradict with the uncertainly principle. However the vanishing normal momentum naturally leads to a symmetry breaking. Thus, if we consider the normal direction as an inner space, we believe that the above requirement is quite natural. In fact our construction is consistent with that in [17], in which (18) was obtained by means of the Dirac constraint quantization scheme under the constraint condition of vanishing momentum.

Further, our study reveals why we need to deform the Hilbert space in the construction of the submanifold quantum mechanics $[8,18]$. If we did not deform the Hilbert space using $\eta_{\mathrm{sa}}, \varpi_{p_{q}}$ can not become a projection operator in the sense of *-algebra [1].

By means of the above construction, we can give a more algebraic representation of generalized Weierstrass relation in terms of the submanifold Dirac operators [24-26,28], which is closely related to the extrinsic Polyakov string [27]. 


\section{References}

[1] Araki H., Mathematical Theory of Quantum Fields, Oxford Univ. Press, Oxford, 1999.

[2] Arfken G., Mathematical Methods for Physicists, Academic Press, New York, 1970.

[3] Björk J-E., Analytic D-Modules and Applications, Kluwer, Dordrecht, 1992.

[4] Burgess C., Goldstone and Pseudo-Goldstone Bosons in Nuclear, Particle and Condensed-Matter Physics, Phys. Rep. 330 (2000) 193-261.

[5] Burgess M. and Jensen B., Fermions Near Two-dimensional Sufraces, Phys. Rev. A 48 (1993) 1861-1866.

[6] Clark I., More on Effective Potentials of Quantum Strip Waveguides, J. Phys. A: Math. Gen. 31 (1998) 2103-2107.

[7] Clark I. and Bracken A., Bound States in Tubular Quantum Waveguides with Torsion, J. Phys. A: Math. Gen. 29 (1996) 4527-4535.

[8] da Costa R., Quantum Mechanics of a Constrained Particle, Phys. Rev. A 23 (1981) 1982-1987.

[9] Dirac P. A. M., The Principles of Quantum Mechanics, Fourth edition, Oxford Univ. Press, Oxford, 1958.

[10] Duclos P., Exner P. and Štovíček P., Curvature-induced Resonances in a Two-dimensional Dirichlet Tube, Ann. Inst. Henri Poincaré 62 (1995) 81-101.

[11] Duclos P., Exner P. and Krejčiřik D., Bound States in Curved Quantum Layers, Commun. Math. Phys. 223 (1995) 13-28.

[12] Encinosa M. and Etemadi B., Surface Distortion Effects on Quantum Dot Helium, Physica B 266 (1999) 361-367.

[13] Exner P. and Šeba P., Bound States in Curved Quantum Waveguides, J. Math. Phys. 30 (1989) 2574-2580.

[14] Goldstone J. and Jaffe R. L., Bound States in Twisting Tubes, Phys. Rev. B 45 (1992) 14100-1407.

[15] Hörmander L., The Analysis of Linear Partial Differential Operators, vol. III, Springer, Berlin, 1985.

[16] Ikegami M. and Nagaoka Y., Quantum Mechanics of Electron on a Curved Interface, Progr. Theor. Phys. Suppl. 106 (1991) 235-248.

[17] Ikegami M., Nagaoka Y., Takagi S. and Tanzawa T., Quantum Mechanics of a Particle on a Curved Surface - Comparision of Three Different Approaches, Progr. Theor. Phys. 88 (1992) 229-249.

[18] Jensen H. and Koppe H., Quantum Mechanics with Constraints, Ann. Phys. 63 (1971) 586-591.

[19] Krejčiřík D., Quantum Strips on Surfaces, J. Geom. Phys. 45 (2003) 203-217. 
[20] Matsutani S., The Physical Meaning of the Embedded Effect in the Quantum Submanifold System, J. Phys. A: Math. Gen. 26 (1993) 51335143.

[21] Matsutani S., The Relation Between the Modified Korteweg-de Vries Equation and Anomaly of Dirac Field on a Thin Elastic Rod, Prog. Theor. Phys. 105 (1994) 1005-1037.

[22] Matsutani S., On the Physical Relation Between the Dirac Equation and the Generalized $m K d V$ Equation on a Thin Elastic Rod, Phys. Lett. A 189 (1994) 27-31.

[23] Matsutani S., Anomaly on a Submanifold System: New Index Theorem Related to a Submanifold System, J. Phys. A: Math. Gen. 28 (1995) 1399-1412.

[24] Matsutani S., Constant Mean Curvature Surface and Dirac Operator, J. Phys. A: Math. Gen. 30 (1997) 4019-4029.

[25] Matsutani S., Immersion Anomaly of Dirac Operator on Surface in $\mathbb{R}^{3}$, Rev. Math. Phys. 11 (1999) 171-186.

[26] Matsutani S., Dirac Operator of a Conformal Surface Immersed in $\mathbb{R}^{4}$ : Further Generalized Weierstrass Relation, Rev. Math. Phys. 12 (2000) 431-444.

[27] Matsutani S., On Density of State of Quantized Willmore Surface :A Way to a Quantized Extrinsic String in $\mathbb{R}^{3}$, J. Phys. A: Math. Gen. 31 (1998) 3595-3606.

[28] Matsutani S., Generalized Weierstrass Relation for a Submanifold $S^{k}$ in $\mathbb{E}^{n}$ Coming from Submanifold Dirac Operator, to appear in Adv. Stud. Pure Math.

[29] Mladenov I., Quantization on Curved Surfaces, I.J.Q.C. 89 (2002) 248-254.

[30] Matsutani S. and Tsuru H., Reflectionless Quantum Wire, J. Phys. Soc. Japan 60 (1991) 3640-3644.

[31] Matsutani S. and Tsuru H., Physical Relation Between Quantum Mechanics and Soliton on a Thin Elastic Rod, Phys. Rev. A 46 (1992) 1144-1147.

[32] Suzuki A. and Matsutani S., Confinement of a Particle in a Ring with Finite Potential, Nuovo Cimento 111 B (1996) 593-606.

[33] Takagi S. and Tanzawa T., Quantum Mechanics of a Particle Confined to a Twisted Ring, Progr. Theor. Phys. 87 (1992) 561-568.

Shigeki Matsutani

8-21-1 Higashi-Linkan

Sagamihara 228-0811

J A P A N

E-mail: RXB01142@nifty.com 